\title{
PARAMETRIC EXTRAPOLATION METHOD FOR DEGENERATE SYSTEM OF LINEAR ALGEBRAIC EQUATIONS ${ }^{1}$
}

\author{
DANG QUANG A
}

Abstract. In this paper we propose an extrapolation method by a spectrum shift parameter for solving degenerate system of linear algebraic equations. An estimate of the computational work for achieving the normal solution with a given accuracy as well as the advantages of the method are shown theoretically and on examples.

Tóm tăt. Trong bài này chúng tôi đề xuất phương pháp ngoại suy theo tham số dịch chuyễn phỗ đễ giải hệ phương trình đại số tuyến tính suy biến, ước lượng khối lượng tính toán cần thiết để đạt được nghiệm chuân tắc với độ chính xác cho trước cũng như tính ưu việt của phương pháp được chỉ ra bằng lý thuyết và bằng các ví dụ.

\section{INTRODUCTION}

In mathematical physics besides boundary value problems with unique solutions we also meet problems having infinite set of solutions, for example, the Neumann problem for elliptic equation. After discretization of this problem by variational methods we get a system of linear algebraic equations (SLAE) with a symmetric, nonnegative matrix. The system usually is nonconsistent because due to the errors of computation of the right-hand side of differential equation the consistence condition may be not satisfied. In order to overcome this defect one introduced the concept of generalized solution and elaborated regularization methods for constructing a stable normal solution (see e.g. [11, 12]). But the problem of estimating computational work for obtaining an approximate solution with a given accuracy has not been considered by researchers. It should be noticed that the authors often consider SLAE without any special structure which arise when processing experimental data.

In this paper we shall treat the system with a symmetric, nonnegative matrix. Our attention will be drawn to the problem of reduction of computational work for getting an approximate normal solution with a given accuracy. The method to be used is the extrapolation technique of solutions of systems with shifted spectrum. This method especially has a great advantage when being performed on parallel computer. The parametric extrapolation technique was used in our earlier works [1-4].

In some sense, this work is a continuation of our previous one [4], where we considered the alternating directions method for solving degenerate system of grid equations.

\section{PRELIMINARIES}

Let us consider the system

$$
A u=f
$$

where $A$ is $n \times n$ matrix, $f \in R^{n}$ and

$$
\operatorname{det} A=0 .
$$

We will regard (2.1) as an operator equation in the space $H=R^{n}$. As usual, we $\operatorname{denote}$ by $\operatorname{Ker} A$ and Image $A$ the kernel and the image of $A$, respectively, and by $A^{*}$ the conjugate operator for $A$. It

* This work was supported in part by the National Basic Program in Natural Sciences, Vietnam. 
is well known that there holds the following decomposition

$$
H=\operatorname{Ker} A^{*} \oplus \operatorname{Im} A .
$$

From(2.3) it follows that the solvability condition of the equation (2.1) in $H$ is

$$
f \perp \operatorname{Ker} A^{*} .
$$

Suppose that $f=\hat{f}+\tilde{f}$, where $\hat{f} \in \operatorname{Im} A, \tilde{f} \in \operatorname{Ker} A^{*}$. Then, if $\tilde{f} \neq 0$ the system (2.1) is nonconsistent.

In this case one introduced the concept of generalized solution.

An element $u \in H$ is called a generalized solution of (2.1) if it satisfies one of the following equivalent problems:

$$
\begin{aligned}
A u & =\hat{f}, \\
A^{*} A u & =A^{*} f, \\
\|A u-f\| & =\min _{v \in H}\|A v-f\| .
\end{aligned}
$$

Generalized solutions of (2.1) always exist and are defined with the accuracy to an element of $K e r A$. The generalized solution of the system (2.1) with minimal norm is called the normal solution of it. This normal solution is unique. Notice that the normal solution of (2.1) is orthogonal to $\operatorname{Ker} A$. For this reason in [10] Tikhonov takes this condition to be the definition of the normal solution of degenerate system.

Now we consider the case when the matrix $A$ is symmetric and nonnegative, i.e. $A=A^{*} \geq 0$, in this case (2.3) become

$$
H=\operatorname{Ker} A \oplus \operatorname{Im} A
$$

and the consistency condition of (2.1) is $f \perp \operatorname{Ker} A$. The Tikhonov regularization method

$$
\min _{u \in H}\left(\|A u-f\|^{2}+\alpha\|u\|^{2}\right)
$$

for finding the normal solution leads to the equation

$$
\left(A^{2}+\alpha I\right) u_{\alpha}=A f
$$

where $I$ is the identity operator.

To solve this SLAE with a given accuracy when $n$ is rather large presents itself a time-consuming work because the spectral range of $A^{2}$ is very large even when the spectral range of $A$ is not very large. Therefore, instead of the usual regularization equation (2.9) for the consistent system (2.1) Tikhonov [10], Fadeeva [5] and Molchanov [7] used the simplified regularization method. Namely, they considered the equation

$$
(A+\alpha I) u_{\alpha}=f .
$$

It is the method of shifting the spectrum of $A$. The necessary and sufficient conditions for regularizing degenerate SLAE by the general shifting spectrum method are presented in [8].

Below we develop the shifting spectrum method in combination with the parametric extrapolation technique in order to reduce the computational amount required for solving the system (2.1).

\section{CASE OF CONSISTENT SYSTEM}

In this section we consider (2.1) under the assumptions that the matrix $A$ is symmetric, degenerate, nonnegative and the consistency condition is satisfied.

Let $e_{1}, e_{2}, \ldots, e_{n}$ be the orthonormal basis of $H$ consisting of the eigenvectors of $A$ and $0=\lambda_{1}=$ $\lambda_{2}=\cdots=\lambda_{m}<\lambda_{m+1} \leq \cdots \leq \lambda_{n}$ be the corresponding eigenvalues. For convenience we denote 
$\lambda_{\min }=\lambda_{m+1}$ and $\lambda_{\max }=\lambda_{n}$. Then we can expand

$$
f=\sum_{i=1}^{n} c_{i} e_{i}
$$

with $c_{i}=\left(f, e_{i}\right)$. Due to the consistency condition we have

$$
c_{i}=0, \quad i=1, \ldots, m \text {. }
$$

We seek the solution of $(2.1)$ in the form

$$
u_{\alpha}=\sum_{i=1}^{n} a_{i}^{(\alpha)} e_{i}
$$

From (2.10) we derive

$$
a_{i}^{(\alpha)}=\frac{c_{i}}{\lambda_{i}+\alpha}, \quad i=1, \ldots, n
$$

Taking into account (3.2) we have

$$
u_{\alpha}=\sum_{i=m+1}^{n} \frac{c_{i}}{\lambda_{i}+\alpha} e_{i}
$$

In the same way we find the normal solution of (2.1)

$$
u^{*}=\sum_{i=m+1}^{n} \frac{c_{i}}{\lambda_{i}} e_{i}
$$

Hence, we have

$$
u_{\alpha}-u^{*}=-\alpha \sum_{i=m+1}^{n} \frac{c_{i}}{\lambda_{i}\left(\lambda_{i}+\alpha\right)} e_{i}
$$

Therefore,

$$
\left\|u_{\alpha}-u^{*}\right\|=\alpha \sqrt{\sum_{i=m+1}^{n} \frac{c_{i}^{2}}{\lambda_{i}^{2}\left(\lambda_{i}+\alpha\right)^{2}}} \leq \frac{\alpha}{\lambda_{\min }}\left\|u^{*}\right\| .
$$

From this estimate it is seen that the deviation of $u_{\alpha}$ from the normal solution $u^{*}$ depends on the chosen regularization parameter $\alpha$ and the smallest positive eigenvalue $\lambda_{\min }$ of $A$. If $\lambda_{\min }$ or certain its estimate is known, then theoretically, the more $\alpha$ is smaller the more accurately $u_{\alpha}$ approximates $u^{*}$. But from the view of computation, when $\alpha$ is too small then condition number of the matrix $A+\alpha I$ is too large and direct solution methods for the system (2.1) on computer may give bad result even run-time error may occur. Also, in this case well-known iterative methods are convergent very slowly even may be not convergent. Therefore, the following question arises: How to find the normal solution with given accuracy spending possibly minimal computational amount? Below this problem will be solved with the help of the parametric extrapolation technique.

Theorem 3.1. For any $k \geq 1$ the solution of the regularized equation (2.10) may be expanded in the form

$$
u_{\alpha}=u^{*}+\sum_{i=1}^{k} \alpha^{i} w_{i}+w_{\lambda} \alpha^{k+1}
$$

where $u^{*}$ is the normal solution of (2.1), $w_{i},(i=1, \ldots, k)$ are elements of $H$ independent of $\alpha$, and

$$
\left\|w_{\lambda}\right\| \leq \frac{\left\|u^{*}\right\|}{\lambda_{\min }^{k+1}}
$$

$\lambda_{\min }$ being the smallest positive eigenvalue of $A$. 
Proof. The proof of the theorem follows directly from (3.5), (3.6) using the Taylor expansion of the function $1 /(\lambda+\alpha)$ in the neighbourhood of the point $\alpha=0$.

Now we put

$$
U^{E}=\sum_{i=1}^{k+1} \gamma_{i} u_{\alpha / i},
$$

where $u_{\alpha / i}$ is the solution of (2.10) with the regularization parameter $\alpha / i$ and

$$
\gamma_{i}=\frac{(-1)^{k+1-i} i^{k+1}}{i !(k+1-i) !}
$$

Using Theorem 3.1 it is easy to show

Theorem 3.2. There holds the estimate

$$
\frac{\left\|U^{E}-u^{*}\right\|}{\left\|u^{*}\right\|} \leq \frac{\alpha^{k+1}}{\lambda_{\min }^{k+1}} .
$$

Remark. In (3.10) taking $k=1$ and

$$
\gamma_{1}=\frac{\alpha_{2}}{\alpha_{2}-\alpha_{1}}, \quad \gamma_{2}=\frac{\alpha_{1}}{\alpha_{1}-\alpha_{2}} .
$$

for two distinct values $\alpha_{1} \neq \alpha_{2}$ we get

$$
U^{E}=\frac{\alpha_{1}}{\alpha_{1}-\alpha_{2}} u_{\alpha_{2}}-\frac{\alpha_{2}}{\alpha_{1}-\alpha_{2}} u_{\alpha_{1}} .
$$

It is the combination which was selected by Fadeeva in [5] although there was not obtained any estimate for error. In the case if the size of the system (2.1) is too large to solve it by direct methods one should use iterative methods (see [9]). Then the gain of the parametric extrapolation in computational amount is great. We show this, for example, for the simple iteration method.

Theorem 3.3. The number of iterations needed for achieving the normal solution of (2.1) with the relative accuracy $\varepsilon$ when applying the simple iteration method to the alone regularized equation (2.10) is

$$
N_{a}=0.5 \frac{\lambda_{\max }}{\lambda_{\min }} \frac{1}{\varepsilon} \ln \frac{1}{\varepsilon}
$$

while this number is

$$
N_{e}=0.25(k+1)(k+2) \frac{\lambda_{\max }}{\lambda_{\min }} \frac{1}{\varepsilon^{1 /(k+1)}} \ln \frac{1}{\varepsilon} .
$$

if using the parametric extrapolation technique (3.10), (3.11). Therefore, the gain of the parametric extrapolation in comparison with the simple spectrum shift method is

$$
G=\frac{2}{(k+1)(k+2)} \frac{1}{\varepsilon^{k /(k+1)}} .
$$

Proof. It is well-known [9] that the number of simple iterations for achieving an approximation $u_{\alpha a}$ for the solution $u_{\alpha}$ of (2.10) with the relative accuracy $\varepsilon$, i.e., $\left\|u_{\alpha a}-u_{\alpha}\right\| /\left\|u_{\alpha}\right\|<\varepsilon$ is

$$
N_{a}=0.5 \frac{1}{\xi} \ln \frac{1}{\varepsilon}
$$

where

$$
\xi=\frac{\alpha}{\lambda_{\max }+\alpha}
$$

From the estimate (3.7) it follows that for $u_{\alpha a}$ approximate $u^{*}$ with the relative accuracy $\varepsilon$ we must choose $\alpha=\varepsilon \lambda_{\min }$. Then we have 


$$
\frac{1}{\xi} \approx \frac{\lambda_{\max }}{\lambda_{\min }} \frac{1}{\varepsilon}
$$

Hence, from (3.17) we get (3.14).

Now, if we construct the extrapolation solution by (3.10), (3.11) then for achieving $U^{E}$ with the relative accuracy $\varepsilon$ we must take $\alpha=\lambda_{\min } \varepsilon^{1 /(k+1}$. With the chosen value of $\alpha$ we can calculate the number of iterations needed for solving (3.10) with the accuracy $\varepsilon$

$$
N=0.5 \frac{\lambda_{\max }}{\lambda_{\min }} \frac{1}{\varepsilon^{1 /(k+1)}} \ln \frac{1}{\varepsilon} .
$$

Therefore, the total number of iterations for $k+1$ regularized equations with the parameters $\alpha, \alpha / 2, \ldots$, $\alpha /(k+1)$ is

$$
N_{e}=(1+2+\ldots+(k+1)) N=0.5 *(k+1)(k+2) N .
$$

Taking into account the expression of $N$ we obtain the formula (3.15). Therefore, the gain of the extrapolation method measured by $G=N_{a} / N_{e}$ will be calculated by (3.16).

Thus, the theorem is proved.

Remark. If using the Chebyshev iterative method instead of the simple one then we get a similar result as stated in Theorem 3.3, where in (3.16) instead of $\varepsilon^{k /(k+1)}$ it should be $\varepsilon^{k / 2(k+1)}$.

By the formula (3.16) we calculated the following table.

\begin{tabular}{|c|c|r|}
\hline$k$ & $\varepsilon$ & \multicolumn{1}{|c|}{$G$} \\
\hline 2 & $10^{-3}$ & 16 \\
2 & $10^{-4}$ & 77 \\
3 & $10^{-4}$ & 100 \\
3 & $10^{-6}$ & 3162 \\
\hline
\end{tabular}

which shows the gain of the extrapolation method.

In the case if the size of the system (2.1) is small direct methods can be used and the computational time is not significant. In this case, using the extrapolation method we can achieve an approximate solution with high accuracy for not too small values of $\alpha$. We show this fact on examples, where we take $k=2$, and therefore, the coefficients $\gamma_{i}$ in (3.10) are $\gamma_{1}=0.5, \gamma_{2}=-4$ and $\gamma_{3}=4.5$. The computation was performed by the software MATLAB 5.3 using the function $u=A \backslash b$ for the solution of $A u=b$ and the long format. The experiment was performed for the regularization parameter $\alpha=10^{-t}, \quad(t=1,2,3,4,5)$. The results are tabulated for the order $m$ of the relative error $\varepsilon=\frac{\left\|u-u^{*}\right\|}{\left\|u^{*}\right\|} \leq 10^{-m}$, where $u=u_{\alpha}, m=m_{a}$ for the simple shifted equation (2.10) and $u=U^{e}, m=m_{e}$ for the extrapolated solution (3.3).

Example 1

$$
A=\left[\begin{array}{ccc}
1 & -1 & 0 \\
-1 & 2 & -1 \\
0 & -1 & 1
\end{array}\right], \quad f=\left[\begin{array}{c}
-1 \\
2 \\
-1
\end{array}\right]
$$

In this case the system (2.1) has the normal solution

$$
u^{*}=(-1 / 3,2 / 3,-1 / 3)^{\prime}
$$

and the results of computation are the following

\begin{tabular}{|c|r|r|r|r|r|}
\hline$t$ & 1 & 2 & 3 & 4 & 5 \\
\hline$m_{s}$ & 1 & 2 & 3 & 4 & 5 \\
\hline$m_{e}$ & 5 & 8 & 11 & 10 & 10 \\
\hline
\end{tabular}


Example 2. Matrix $A=\left(a_{i j}\right)$ is of the sizes $11 \times 11$ and as the same tridiagonal structure as in Example 1, namely,

$$
\begin{aligned}
& a_{i i}= \begin{cases}1, & i=1,11 \\
2, & \text { otherwise }\end{cases} \\
& a_{i, i+1}=-1, \quad i=1, \ldots, 10 \\
& a_{i-1, i}=-1, \quad i=2, \ldots, 11 \\
& a_{i, j}=0, \quad \text { otherwise } \\
& f=\left(f_{i}\right), \quad f_{i}= \begin{cases}-1, & i=1,11 \\
2 & \text { otherwise }\end{cases}
\end{aligned}
$$

In this case the system (2.1) has the normal solution

$$
u^{*}=(15,15,14,12,9,5,0,-6,-13,-21,-30)^{\prime}
$$

and the result of computation is the following

\begin{tabular}{|c|c|c|c|c|c|}
\hline$t$ & 1 & 2 & 3 & 4 & 5 \\
\hline$t$ & 1 & 2 & 3 & 4 & 5 \\
\hline$m_{s}$ & 0 & 1 & 2 & 3 & 4 \\
\hline$m_{e}$ & 1 & 4 & 7 & 10 & 11 \\
\hline
\end{tabular}

\section{CASE OF NONCONSISTENT SYSTEM}

In this section we also assume that the matrix $A$ is symmetric, degenerate, nonnegative but the system is not consistent. In this case it is easy to verify that the normal solution of (2.1) also is

$$
u^{*}=\sum_{i=m+1}^{n} \frac{c_{i}}{\lambda_{i}} e_{i}
$$

but the solution of the equation (2.10) is

$$
u_{\alpha}=\sum_{i=1}^{m} \frac{c_{i}}{\alpha} e_{i}+\sum_{i=m+1}^{n} \frac{c_{i}}{\lambda_{i}+\alpha} e_{i} .
$$

Notice that $u_{\alpha}$ can not be regarded as an approximate of the normal solution $u^{*}$. As in Section 3 it is easy to prove the following

Theorem 4.1. For any $k \geq 2$ the solution of the regularized equation (2.10) may be expanded in the form

$$
u_{\alpha}=u^{*}+\frac{1}{\alpha} \tilde{f}+\sum_{i=1}^{k-1} \alpha^{i} w_{i}+w_{\lambda} \alpha^{k}
$$

where $u^{*}$ is the normal solution of (2.1), $\tilde{f}$ is the projection of $f$ onto $\operatorname{Ker} A, w_{i},(i=1, \ldots, k-1)$ are elements of $H$ independent of $\alpha$, and

$$
\left\|w_{\lambda}\right\| \leq \frac{\left\|u^{*}\right\|}{\lambda_{\min }^{k}},
$$

$\lambda_{\min }$ being the smallest positive eigenvalue of $A$.

Now, suppose $\alpha_{1}, \alpha_{2}, \ldots, \alpha_{k+1}$ are distinct positive numbers. Consider the following system for $\gamma_{1}, \gamma_{2}, \ldots, \gamma_{k+1}$ 


$$
\begin{aligned}
& \sum_{j=1}^{k+1} \frac{\gamma_{j}}{\alpha_{j}}=0, \\
& \sum_{j=1}^{k+1} \gamma_{j}=1, \\
& \sum_{j=1}^{k+1} \alpha_{j}^{l} \gamma_{j}=0, \quad l=1,2, \ldots, k-1 .
\end{aligned}
$$

It is possible to verify the following

Lemma. The system (4.5) has a unique solution

$$
\gamma_{l}=-\frac{\sum_{j \neq l} \frac{1}{\alpha_{j}} \prod_{i=1}^{k+1} \alpha_{i}}{\prod_{j \neq l}\left(\alpha_{j}-\alpha_{l}\right)} .
$$

In the particular case, when $\alpha_{j}=\alpha / j \quad(j=1,2, \ldots, k+1)$ we have

$$
\gamma_{l}=(-1)^{k+l}\left(\frac{(k+1)(k+2)}{2}-l\right) \frac{l^{k}}{l !(k+1-l) !} .
$$

From the above lemma and Theorem 4.1 we get the following

Theorem 4.2. Let $\gamma_{l} \quad(l=1,2, \ldots, k+1)$ be given by the formula (4.7). Then for the extrapolation solution

$$
U^{E}=\sum_{i=1}^{k+1} \gamma_{i} u_{\alpha / i}
$$

where $u_{\alpha / i}$ is the solution of (2.10) with the regularization parameter $\alpha / i$ we have the estimate

$$
\frac{\left\|U^{E}-u^{*}\right\|}{\left\|u^{*}\right\|} \leq \frac{\alpha^{k}}{\lambda_{\min }^{k}}
$$

Note that in the case if the system (2.1) is not consistent then the combination of $k+1$ solutions of (2.10) with parameters $\alpha / j$ approximates the normal solution $u^{*}$ only with the accuracy of order $\alpha^{k}$. It completely fits to the fact mentioned above that an alone solution of (2.1) does not give an approximation to $u^{*}$. Analogously as in Section 3 we have the following estimate of computational amount for getting the normal solution with a given accuracy.

Theorem 4.3. The number of iterations needed for achieving the normal solution of the nonconsistent system (2.1) with the relative accuracy $\varepsilon$ by the extrapolation method when applying the simple iteration method to each of the $k+1$ system with shifted spectrum (2.10) is

$$
N_{e}=0.25(k+1)(k+2) \frac{\lambda_{\max }}{\lambda_{\min }} \frac{1}{\varepsilon^{1 / k}} \ln \frac{1}{\varepsilon}
$$

\section{REFERENCES}

[1] Dang Quang A, Approximate method for solving an elliptic problem with discontinuous coefficients, Journal of Comput. and Appl. Math. 51 (1994) 193-203. 
[2] Dang Quang A, Mixed boundary-domain operator method in approximate solution of biharmonic type equation, Vietnam Journal of Math. 26 (1998) 243-252.

[3] Dang Quang A, Construction of iterative methods for solving a mixed boundary value problem for biharmonic equation, Proceedings of the Fifth Vietnamese Mathematical Conference, 1997, Sci. and Tech. Publ. House, Hanoi, 1999, 47-55.

[4] Dang Quang A, Iterative methods for solving degenerate system of grid equations I, Journal of Comput. Sci. and Cyber. 13 (4) (1997) 33-45.

[5] Fadeeva V.N., Shift for system with ill-conditioned matrices, J. of Comp. Math. And Math. Phys. 5 (1965) 907-911 (Russian).

[6] Marchuk G.I., Methods of Numerical Mathematics, Nauka, Moscow, 1989 (Russian).

[7] Molchanov I., Numerical Methods for Some Problems of Elasticity Theory, Kiev, Naukova Dumka, 1979 (Russian).

[8] Morozov V.A. and Nazimov A.B., About necessary and sufficient conditions for regularizing degenerate system of linear algebraic equations by the shifting spectrum method, J. of Comp. Math. And Math. Phys. 26 (1986) 1283-1290 (Russian).

[9] Samarskij A. and Nikolaev E., Numerical Methods for Grid Equations, Vol. 2, Birkhauser, Basel, 1989.

[10] Tikhonov A.N., About the stability of algorithms for solving degenerate system of linear algebraic equations, J. of Comp. Math. And Math. Phys. 5 (1965) 718-722 (Russian).

[11] Tikhonov A. and Arsenin V., Solutions of Ill-posed Problems, Wiley, New York, 1977.

[12] Tikhonov A. N., Goncharsky A. V., Stepanov V.V., Yagola A. G., Numerical Methods for the Solution of Ill-posed Problems, Dordrecht - Kluwer Acad. Publ., 1995.

Received December 7, 2000 Revised January 15, 2002

Institute of Information Technology 